\title{
Comparative Simulation Studies of Native and Single Site Mutant Human Beta-Defensin-1 Peptides
}

\author{
Supplementary Material \\ Rabab A. Toubar, ${ }^{\dagger \dagger}$ Artem Zhmurov, ${ }^{\dagger}$ Valeri Barsegov, ${ }^{\dagger *}$ and Kenneth A. Marx ${ }^{\dagger *}$ \\ ${ }^{\dagger}$ Department of Chemistry, University of Massachusetts Lowell, MA 01854; ${ }^{\ddagger}$ Pharmaceutical \\ Chemistry Department, Faculty of Pharmacy, Ain Shams University, Abbassia, Cairo 11566, Egypt. \\ *Corresponding authors: Valeri_Barsegov@uml.edu or Kenneth_Marx@uml.edu
}

Keywords: Human beta defensin-1, Molecular Dynamics simulations

Running Title: Native and Single Site Mutant Human Beta-Defensin-1 Simulations 


\section{TABLES}

Table SM1: Details of MD simulations including: PDB codes, mutation sites, trajectory lengths, and the number of simulation runs for all the systems. Monomers and dimers were used in equilibrium simulations at $T=300 \mathrm{~K}$; heating simulations (from $T_{i n}=300 \mathrm{~K}$ to $T_{\text {fin }}=500 \mathrm{~K}$ ) were carried out only for the monomeric species.

\begin{tabular}{|c|c|c|c|c|}
\hline System & $\begin{array}{c}\text { PDB } \\
\text { code }\end{array}$ & $\begin{array}{c}\text { Mutation } \\
\text { site }\end{array}$ & $\begin{array}{c}\text { Equilibrium } \\
\text { Simulations }\end{array}$ & $\begin{array}{c}\text { Heating } \\
\text { Simulations }\end{array}$ \\
\hline WT monomer & $1 \mathrm{IJV}$ & - & $30 \mathrm{~ns}$ (3 runs) & $10 \mathrm{~ns}$ \\
\hline WT monomer & $1 \mathrm{IJV}$ & - & $25 \mathrm{~ns}(3 \mathrm{runs})^{\S}$ & - \\
\hline Mut1 monomer & $2 \mathrm{NLB}$ & Asn4Ala & $30 \mathrm{~ns}$ (3 runs) & $10 \mathrm{~ns}$ \\
\hline Mut2 monomer & $2 \mathrm{NLP}$ & Gln24Glu & $30 \mathrm{~ns}$ (3 runs) & $10 \mathrm{~ns}$ \\
\hline Mut3 monomer & $2 \mathrm{NLQ}$ & Lys31Ala & $30 \mathrm{~ns}$ (3 runs) & $10 \mathrm{~ns}$ \\
\hline WT dimer & $1 \mathrm{IJV}$ & - & $30 \mathrm{~ns}$ (3 runs) & - \\
\hline Mut1 dimer & $1 \mathrm{IJV}^{*}$ & Asn4Ala & $30 \mathrm{~ns}$ (3 runs) & - \\
\hline Mut2 dimer & $1 \mathrm{IJV}^{*}$ & Gln24Glu & $30 \mathrm{~ns}$ (3 runs) & - \\
\hline Mut3 dimer & $1 \mathrm{IJV}^{*}$ & Lys31Ala & $30 \mathrm{~ns}(3$ runs) & - \\
\hline
\end{tabular}

${ }^{\S}$ GROMACS simulations: three runs (a, b, and c) were performed using three different force fields (see, also, Fig. SI3).

*Due to the absence of mutant dimers in the PDB, 1IJV (WT) dimers were mutated at the reported mutation site (see main text). 

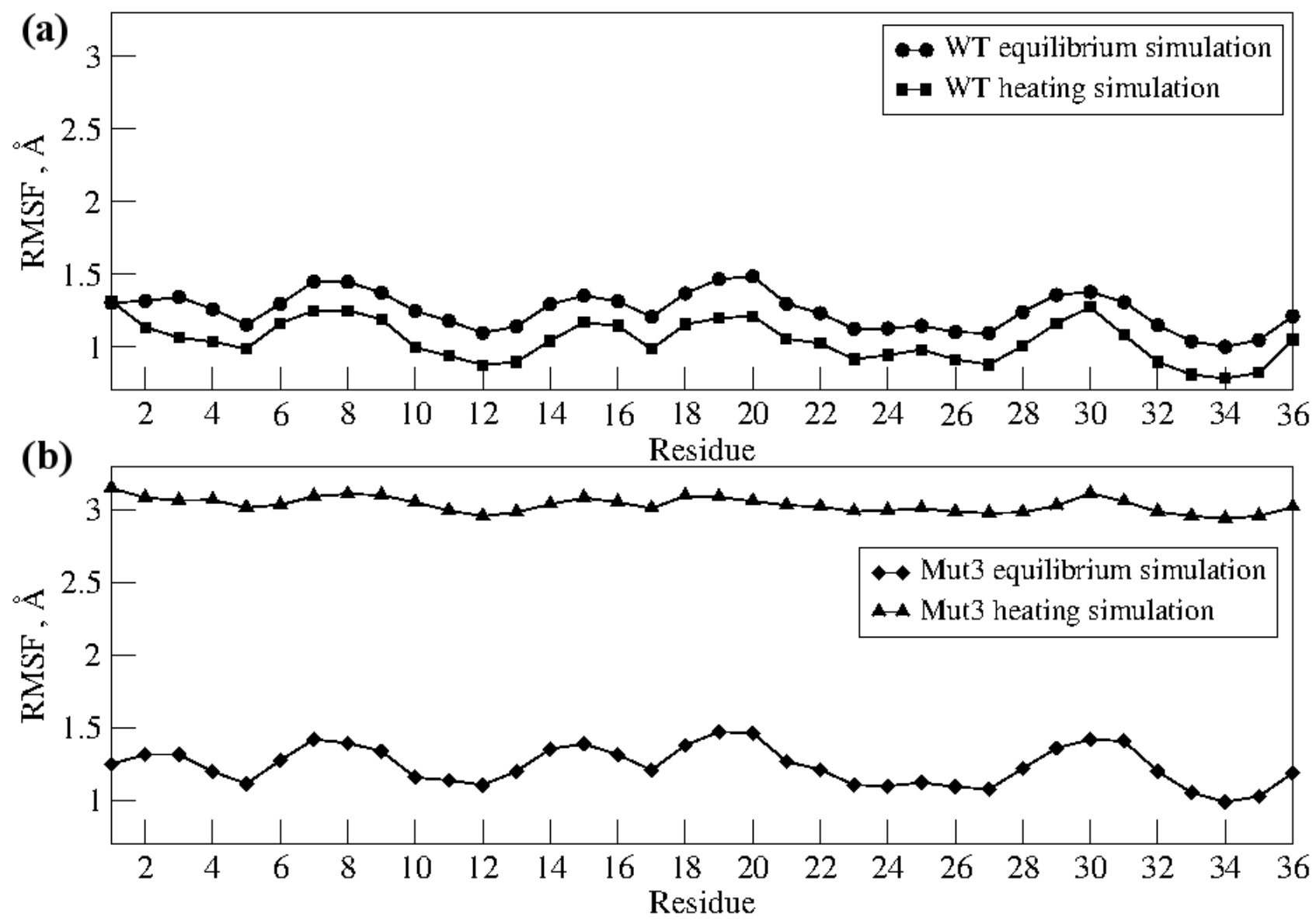

Fig. SM1: The root-mean-square fluctuations (RMSF) for the monomers of native HBD-1 peptide (WT) (panel (a)), and Mut3 (panel (b)) obtained from averaging over three equilibrium simulation runs at room temperature $T=300 \mathrm{~K}$ (WT; dots and Mut3; diamonds), and from heating simulations at increasing temperature (from $T_{i n}=300 \mathrm{~K}$ to $T_{\text {fin }}=500 \mathrm{~K}$ : WT; squares and Mut3; triangles). In panel (a), the RMSF curve for heating simulation is below the RMSF curve for WT, whereas in panel (b) the RMSF curve for heating simulation of Mut3 lies above the RMSF curve for equilibrium simulations. 
WT
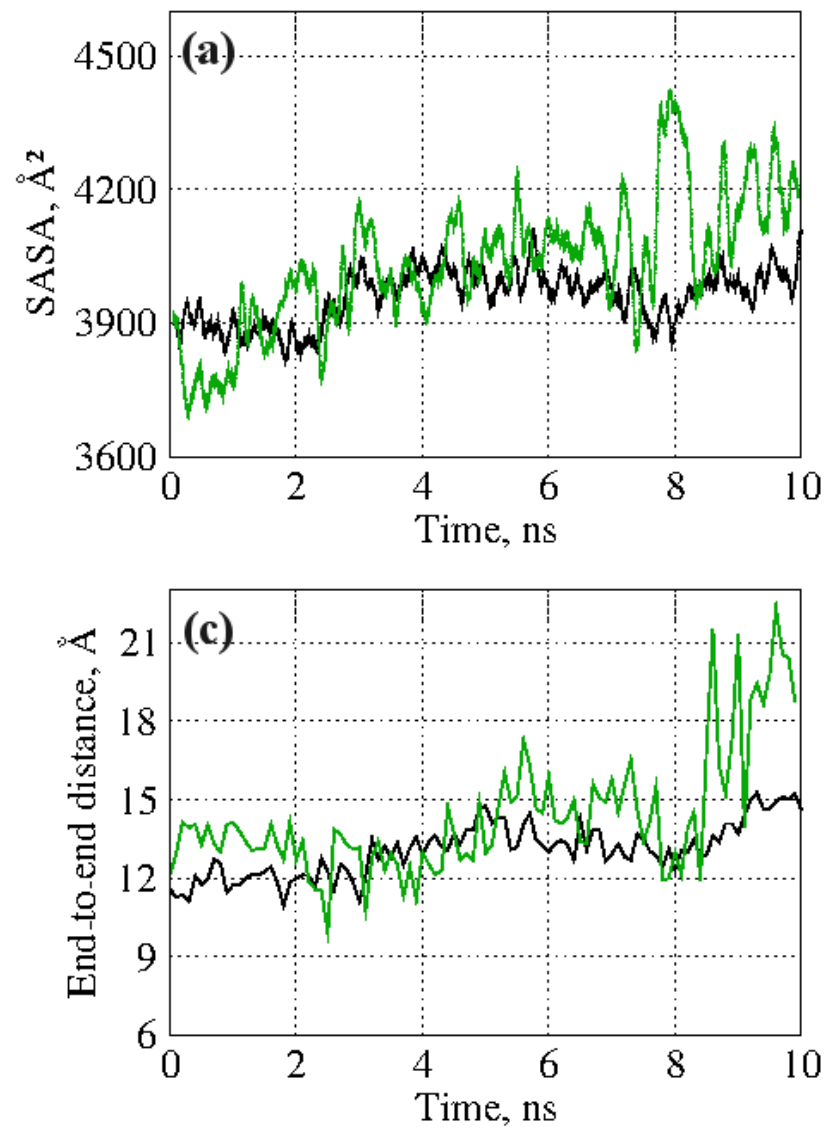

Mut2
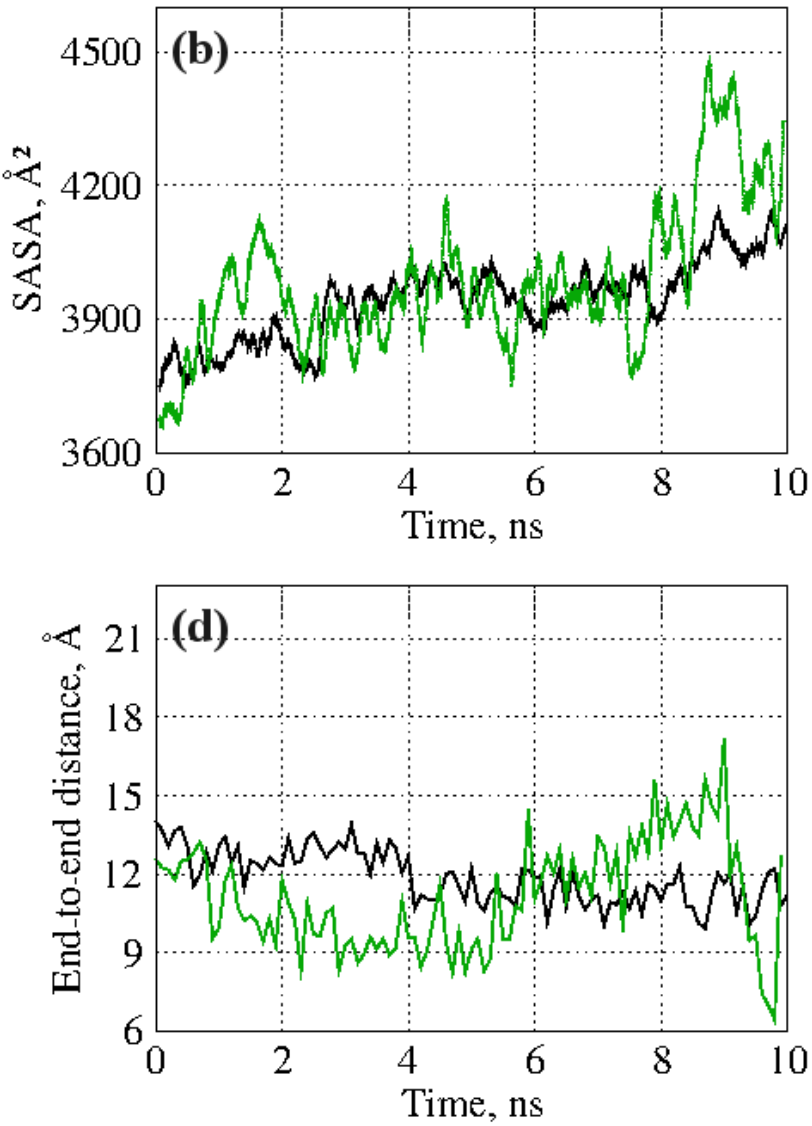

Fig. SM2: Panels (a) and (b): The total solvent accessible surface area (SASA) versus time for the monomers of native HBD-1 peptide (WT) (panel (a)) and Mut2 (panel (b)). Panels (c) and (d): The time dependence of the end-to-end distance for the monomers of WT (panel (c)) and Mut2 (panel (d)). Plots were obtained from $30 \mathrm{~ns}$ equilibrium simulation runs at $T=300 \mathrm{~K}$ (average of three trajectories; black curves), and from heating simulations at increasing temperature (from $T_{\text {in }}=300 \mathrm{~K}$ to $T_{\text {fin }}=500 \mathrm{~K}$; green curves). Equilibrium simulations curves were truncated at $10 \mathrm{~ns}$ due to only minor differences observed beyond that point. 


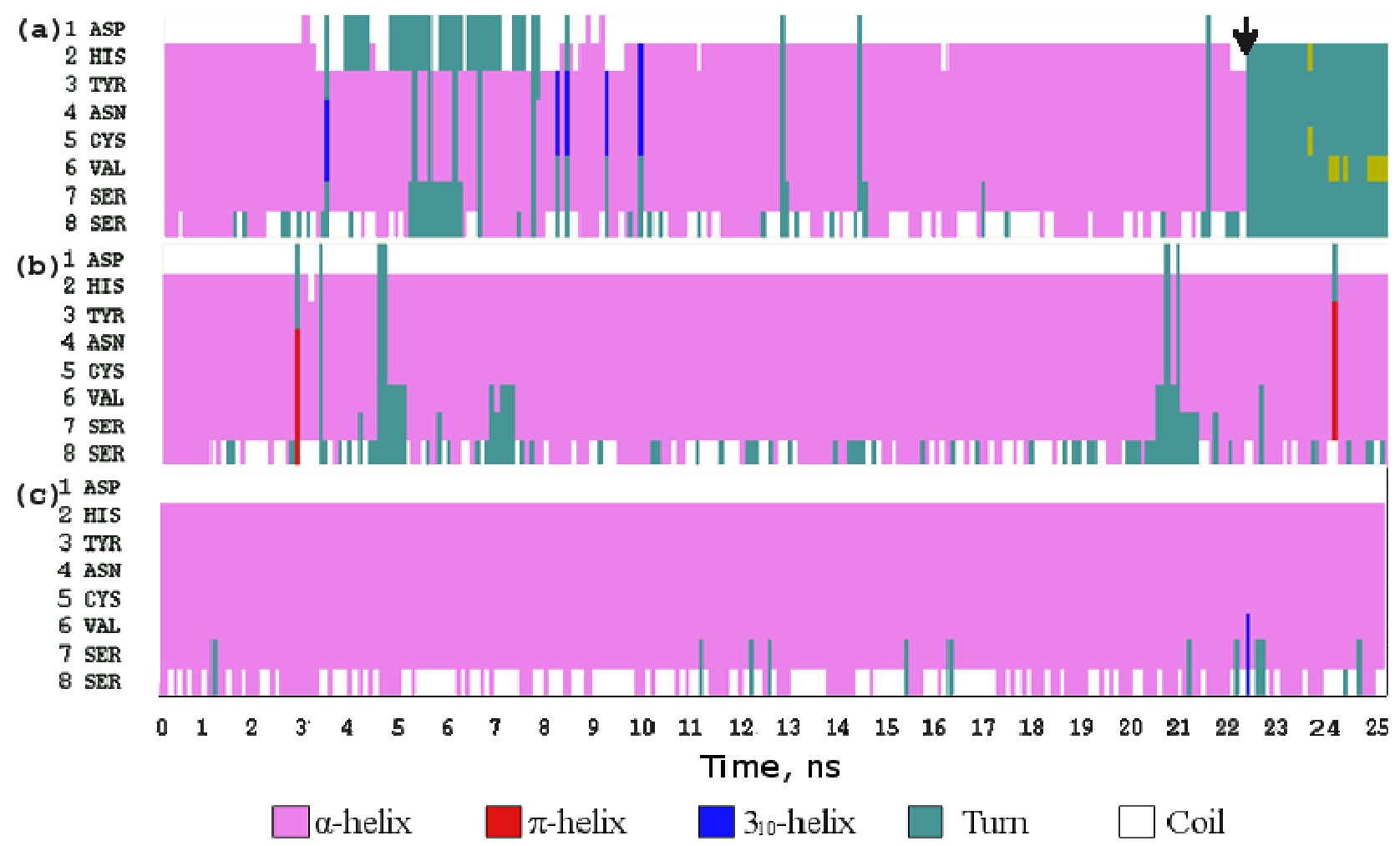

Fig. SM3: Time evolution of the secondary structure propensity for the $\alpha$-helical portion (residues 27) of the native HBD-1 peptide (WT) monomer from equilibrium simulations at $T=300 \mathrm{~K}$ using the GROMACS software package applying GROMOS force field and twin range cutoff (panel (a)), GROMOS and Particle Mesh Ewald (PME) summation method (panel (b)), and OPLS AA force field and PME (panel (c)). Minor $\pi$-helix occurrence was observed when compared to simulation results obtained using the NAMD package applying CHARMM force field (Fig. 2 in the main text). In panel (a), the arrow at $22.5 \mathrm{~ns}$ indicates the point when $\alpha$-helix became unstructured. Color denotation is presented in the graph. 